\title{
Protée
}

\section{De la texture à la matière}

\section{Anne Beyaert-Geslin}

Volume 36, numéro 2, automne 2008

Éthique et sémiotique du sujet

URI : https://id.erudit.org/iderudit/019026ar

DOI : https://doi.org/10.7202/019026ar

Aller au sommaire du numéro

\section{Éditeur(s)}

Département des arts et lettres - Université du Québec à Chicoutimi

\section{ISSN}

0300-3523 (imprimé)

1708-2307 (numérique)

Découvrir la revue

\section{Citer cet article}

Beyaert-Geslin, A. (2008). De la texture à la matière. Protée, 36(2), 101-110.

https://doi.org/10.7202/019026ar

\section{Résumé de l'article}

Si la sémiotique a montré l'importance de la texture dans la signification, la dimension de la matière a été largement négligée. L'article s'efforce de distinguer ces deux dimensions et de caractériser la matière de façon à en montrer l'intérêt pour la sémiotique. En première approximation, la texture se laisse concevoir relativement à la pratique de la peinture alors que la matière nous situe généralement en dehors des pratiques ; mais, en certains cas, elle peut caractériser la peinture et décrit alors une épaisseur du tableau, une tridimensionnalité. Nous partirons de ce constat pour décrire, au travers des oeuvres de Joseph Beuys, la matière comme un actant pouvant régir, au-delà de la forme énoncée, la signification dans son acception la plus large. Décrire ces oeuvres telles des natures mortes nous permettra de montrer que la matière est une structure narrative imposant dès l'abord une axiologie.
Ce document est protégé par la loi sur le droit d'auteur. L’utilisation des services d'Érudit (y compris la reproduction) est assujettie à sa politique d'utilisation que vous pouvez consulter en ligne.

https://apropos.erudit.org/fr/usagers/politique-dutilisation/ 


\title{
DE LA TEXTURE À LA MATIÈRE
}

\author{
ANNE BEYAERT-GESLIN
}

Si la sémiotique s'est constituée en une discipline autonome en se consacrant à la textualité, elle a cependant intégré peu à peu une instance corporelle et envisagé progressivement la réception dans son rapport au corps de l'observateur considéré comme instance de conceptualisation et de stabilisation de la semiosis. En sémiotique visuelle, cette prise en compte s'est engagée à partir de la dimension plastique (Floch, 1985) et de la texture pour autant que celle-ci renvoie à "la trace du geste» (Groupe $\mu, 1992$ : 195) et témoigne à la fois de l'intervention du corps de l'énonciateur, par cette entremise, et des propriétés du support. Sur cette base, le Groupe $\mu$ aborde donc la texture à partir de trois instances - le support, la matière et la manière qui permettent de fixer différentes «familles d'unités texturales» (ibid.: 203).

Si cette conception constitue une avancée importante, spécialement parce qu'elle donne toute autorité à la texture pour assurer la distinction figure-fond, la catégorisation et donc la distribution des valeurs, elle ménage cependant un certain nombre de points de discussion. En effet, bien que le premier rôle lui soit attribué dans l'énonciation, la texture reste, selon cette conception, dépendante d'un support et du geste $d^{\prime}$ énonciation (la manière du Groupe $\mu$ ) et afférente à la pratique picturale. Or, des œuvres relevant d'autres pratiques permettent de s'acquitter du support, ce qui conduit à reconsidérer les propriétés de la matière, désormais investie de compétences supplémentaires.

Alter ego du corps dans l'énonciation, la matière ainsi envisagée contient déjà toutes les potentialités de la textualité et en anticipe la forme, de sorte que la praxis énonciative se laisse décrire comme une actualisation de ces propriétés, une actua- lisation de la matière. Plus exactement, la matière est traversée par une direction intentionnelle qui suffit à lui conférer un statut actantiel, orientant ainsi une semiosis que la praxis énonciative viendra stabiliser.

Afin de valider cette hypothèse actantielle, nous proposons de distinguer la texture, propriété de la surface résultant de la coopération des trois instances (support, matière et manière, pour le Groupe $\mu$ ), et la matière elle-même, instance autonome animée d'un principe actantiel se manifestant au plan de l'expression par cette propriété de surface. À partir de différents exemples de peinture, nous discuterons le rapport de la texture et du support, avant d'aborder la matière, en nous plaçant hors de la pratique picturale avec des œuvres de Joseph Beuys. Nous verrons alors que cette dernière se prête à une description non seulement objective mais également narrative, voire passionnelle, prévoyant des conflits de modalités et des séquences programmées. La matière est alors un acteur investi d'une moralité.

\section{La texture des peintres}

Parce qu'ils adoptent résolument le point de vue de la pratique, les peintres ont toujours considéré l'énonciation comme une association d'instances coopérant à la production du tableau. Parmi bien d'autres artistes, Dubuffet s'est efforcé de rendre compte de cette coopération: "L'art doit naître du matériau et de l'outil et doit garder la trace de l'outil et de la lutte de l'outil avec le matériau. L'homme doit parler mais I'outil aussi et le matériau aussi» (1973: 25). Selon lui, I'artiste doit suivre son matériau, "chaque matériau ayant son langage, étant un langage, qui n'en admet aucun autre et ne peut en servir aucun» (ibid.: 23). 
Un tel commentaire semble de prime abord exemplaire, car, faisant le lien entre le faire de l'artiste et les propriétés du support, il décrit la texture telle une trace, mais, surtout, parce qu'il témoigne de l'utilisation du mot matériau par la pratique picturale. Cet usage trouverait écho chez de nombreux peintres, par exemple chez Moholy-Nagy qui, dans une même page de ses écrits 1 , donne à la matière un sens général dont témoigne l'absence de spécification, tandis qu'il associe le matériau à des usages précis, spécifiés par un qualificatif, tel que pictural ou de construction (à propos du béton, de l'acier, etc.).

Ces premiers apports semblent situer la différence entre la matière et le matériau relativement à la pratique sémiotique qui, se trouvant elle-même caractérisée par une finalité, tend à finaliser la matière pour en faire un matériau. Matière finalisée ${ }^{2}$, un matériau est nécessairement associé à une pratique; il entre dans un programme d'action précis dont il prédit la scène prédicative et le modus operandi. Ainsi, dans la mesure où la matière utilisée par la pratique picturale (autrement dit le matériau pictural) est un pigment se présentant sous forme de poudre, un liquide ou une pâte selon qu'un liant est requis, elle doit être "appliquée», ce qui amène Dubuffet à décrire en creux l'instance du support ainsi qu'un principe d'énonciation par recouvrement de ce support. Ce modus operandi invariable admet pourtant d'infinies variantes en fonction des propriétés des instances en présence:

La même poudre d'outremer prendra une infinité d'aspects différents selon qu'elle sera mêlée d'huile ou d'œuf, ou de lait, ou de gomme. Et qu'ensuite, elle sera appliquée sur du plâtre, sur du bois, sur du carton ou sur une toile (et naturellement, suivant quelle toile et sa préparation). Lissée ou non. Plus ou moins opaque.

(Dubuffet, 1973: 26)

Cette conception, qui décrit le support sur un principe de recouvrement en associant l'énonciation à des couches de couleur, illustre le principe génératif de la peinture qui procède toujours par ajout (addition) contrairement à la sculpture, par exemple, qui peut opter pour l'addition ou la soustraction, comme I'indique Klee (1995: 95), une alternative qu'incarne l'œuvre de Brancusi notamment (Beyaert-Geslin, 2003a: 78). Le principe génératif du recouvrement fait ainsi pendant à une conception du support procédant par inscription et convenant à la pratique scripturale ${ }^{3}$. Des nuances devraient sans doute être apportées, par exemple pour envisager le cas où la peinture appliquée sur un support devient elle-même support pour des couches supplémentaires, comme dans les tableaux d'Eugène Leroy dans lesquels le recouvrement permet de dissimuler la figure initiale sous d'épaisses couches d'où elle émerge seulement sous forme de points de couleur. En pareille situation énonciative, une «chair de la peinture» se confond alors avec la chair du monde, à la manière du Bœuf écorché de Rembrandt.

Une autre précaution conduirait à envisager un terme complexe entre le support de recouvrement de la peinture et le support d'inscription de l'écriture: le cas de la fresque. Bien qu'appliquée avec les outils du peintre (pinceaux et brosses), la couleur ne forme pas des couches mais se trouve cristallisée dans le mortier frais dont le mur est enduit. Cette pratique spécifique, qui exige un geste extrêmement sûr et n'admet aucun repentir de la part du peintre, révèle une tension entre le recouvrement et I'inscription, entre le sur et le dans, qui confronte la pratique picturale à celle de l'écriture. Il serait sans doute intéressant de prospecter dans cette voie pour envisager d'autres rapports singuliers entre le matériau pictural et le support qui mettent en difficulté le principe génératif de la texture.

\section{Support matériel et support formel}

Le rapport du matériau pictural à son support se laisse décrire à l'aune d'une tension historique entre la matérialisation et la dématérialisation. En peinture, la prise en compte des propriétés matérielles par la semiosis s'incarne de multiples façons, mais trouve une formulation exemplaire chez Rothko, où l'extrême délicatesse du support matériel apporte sa contribution à I'aspect poudreux de la peinture, donc à la semiosis ${ }^{4}$. À l'inverse, la tension dématérialisante s'accorde avec une pression iconisante et un projet figuratif centré sur la description des objets du monde. Dans son effort pour relier/différencier les projets de la peinture, de la photographie et du film, Moholy-Nagy rend compte d'une telle dématérialisation lorsqu'il avance que, depuis l'invention de la loi de la perspective, le tableau s'est «presque exclusivement concentré sur la représentation» (2007: 91). Là encore, de multiples exemples sont possibles, dans des registres très divers allant de la Figuration narrative à Magritte qui, dans son effort pour décrire les objets du monde, les isoler et les intégrer à de nouvelles configurations, semble s'acquitter des propriétés du support matériel du tableau. Mais une telle affirmation doit être nuancée car, si ces tensions s'exercent indiscutablement, la dématérialisation n'entraîne jamais la «liquidation » du support matériel. Cette adhérence du support nous permettra de discuter le concept d'image.

Observons la peinture de Magritte. À aucun moment, I'apparence de ses tableaux ne se confond avec celle d'une gravure ou d'une photographie, par exemple. Loin d'être imputable à la 
maladresse, la fausse gaucherie de certaines formes indique que I'effort du peintre n'est pas tant de satisfaire une parfaite ressemblance iconique que d'assurer l'identification des objets du monde, qui seront ainsi rapportés à leur représentation picturale. Cela nous amènerait à constater que ces tableaux assument, au travers de la texture et de l'eidétique, un métadiscours sur la peinture. Loin de nous égarer, cet exemple permet de postuler que la spécificité de la peinture impose une connivence du geste et du support impliqués dans la praxis énonciative. Les inférences de cette «adhérence du support » se laissent mieux décrire à partir d'une catégorie mise en œuvre à propos de l'écriture (Fontanille, 2005), qui oppose le support matériel au support formel. Cette catégorie se fonde sur le principe d'une actualisation des propriétés du support matériel dans la matérialisation; avec la dématérialisation, ces propriétés sont au contraire potentialisées et synthétisées dans ce qui se laisse alors décrire comme un support formel. Si l'on conçoit aisément que le support matériel puisse apporter sa contribution à la semiosis lorsque ses propriétés sont actualisées, il resterait à décrire l'incidence de leur potentialisation dans le support formel, leur participation à la signification. Or, l'exemple des tableaux de Magritte suggère précisément une « résistance» du support matériel qui, loin de disparaître, se transforme en support formel tandis que ses propriétés se trouvent transférées, synthétisées et mises en mémoire. Ainsi le mouvement matérialisation/dématérialisation se trouve-t-il nuancé et mesuré par la transformation du support matériel en support formel.

Mais il semble intéressant de creuser ce point pour caractériser la différence entre le tableau et l'image. Un énoncé visuel qui s'acquitte du support matériel pour en condenser les propriétés dans un support formel est susceptible de migrer d'un support à I'autre sans que sa signification n'en soit jamais modifiée. Il devient alors une image, cette instance globalisante de la sémiotique visuelle susceptible $d^{\prime}$ accueillir tous les énoncés parce qu'elle virtualise, dès l'abord, toutes les spécificités des pratiques. Pour le sémioticien, étudier l'image plutôt que le tableau ou la photographie revient donc à se placer en dehors des pratiques. Ce point de vue épistémologique, commode, permet de constituer des corpus en réunissant les énoncés visuels les plus variés sur une ressemblance eidétique (des affiches électorales, des portraits peints et des médaillons, par exemple) ${ }^{5}$, mais reste critiquable parce qu'il sacrifie un certain nombre de participations essentielles, et en premier lieu l'alliance du support, du geste et de la matière dans la texture qui, en tant que spécificité de la peinture, apporte pourtant une contribution majeure à la signification.
Cependant, méditer l'exemple de Magritte amènerait à convenir que ses tableaux ne se réduisent jamais au statut d'image parce que leur signification reste - fût-ce lorsqu'ils ornent des produits dérivés, papier à lettre ou tapis de souris - marquée par la pratique picturale: ce ne sont pas des images mais des images de tableaux, voire, parce que des connotations y apportent leur contribution, des images de tableaux de Magritte. Si ces «énoncés migrateurs » ont en commun de transporter le support matériel du tableau consigné dans un support formelà peu près constant ${ }^{6}$, une telle ressemblance ne doit cependant pas occulter des différences essentielles qui tiennent au fait que chaque occurrence des images de tableaux de Magritte renouvelle la situation énonciative, le rapport au corps de l'observateur s'avérant très différent lorsqu' « un Magritte» orne un tapis de souris, un médaillon ou une tasse à café.

L'éventuelle généralisation de ces remarques à d'autres corpus de peinture permettrait en tout cas de valider une particularité de la représentation diversement discutée par Goodman (1968: 58) et Greenberg (1989: 12-13)7. En effet, si une représentation décrit le monde en même temps qu'un certain rapport au monde - une thèse majeure de Goodman -, une peinture doit être tenue à la fois pour un discours et pour un métadiscours par lequel elle se commente elle-même. Se manifestant nécessairement sur un mode réflexif, en tant qu'image $d^{\prime}$ 'une peinture, elle conserve en mémoire les propriétés du support matériel dans le support formel. Élargissant l'approche et gardant à l'esprit à la fois les tensions matérialisante/dématérialisante et la leçon de Goodman, nous pourrions avancer qu'une représentation peut certes tendre vers I'image lorsqu'elle est reproduite, être considérée comme telle, mais qu'une mémoire matérielle du support est nécessairement conservée, qui en fait une image de... et témoigne de l'alliance du geste et du support dans la texture, d'un modus operandi et d'une pratique particulière.

\section{La matière et le matériau pictural}

Si elles permettent de caractériser le rapport du support et du matériau pictural, ces réflexions ne donnent pas accès à la matière envisagée dans sa généralité définitoire. Or, si la peinture recourt au matériau pictural pour qualifier la couleur, le terme de matière est également admissible en certains cas précis qui autorisent la qualification de matiériste, comme chez Tapiès ou Dubuffet. Dans ces tableaux, la matière alimente pour ainsi dire le matériau dont elle adopte le principe génératif de la couche. Elle ajoute donc, conformément au principe du recouvrement afférent à la pratique picturale, sorte d'ajout à l'ajout, en pro- 
cédant à une désorganisation/réorganisation de la texture dont il semble utile de préciser les enjeux.

Le Groupe $\mu$ (1992 : 197-199) classe les «familles texturales » selon deux paramètres dits texturèmes: le choix d'un élément et sa loi de répétition. II note qu'un mouvement d'oscillation du regard permet d'intégrer les éléments à la continuité de la forme peinte (intégration) ou de les en "extraire», la sommation occasionnant une désintégration de la forme. Cette description laisse penser que l'intégration d'une matière hétérogène viendrait perturber le mouvement alternatif du regard en confrontant la discontinuité des applications ponctuelles à la continuité et à la régularité qui caractérisent la texture ${ }^{8}$, induisant ainsi un affaiblissement de la texture, dont la régularité est compromise.

Mais une telle description ne saurait suffire. En effet, la matière ajoutée se laissant elle aussi décrire à l'aune des paramètres du Groupe $\mu$ (le choix des éléments et leur régularité) ces deux critères donnant la pleine mesure de l'innovation artistique (il s'agit de désigner de nouvelles matières comme matériau pictural, qui susciteront d'autres modes d'application initiateurs de nouvelles régularités) -, l'intervention ne se laisse pas limiter à la simple désorganisation mais se conçoit comme une nouvelle énonciation du tableau entraînant un réagencement complet du sens. L'histoire de l'art du xxe siècle révèle maints exemples d'utilisation innovante de la matière (poussières, sable, apprêts, filets, voire objets manufacturés) où le principe du recouvrement fait place au collage d'éléments fluides ou solides (les poussières de Tapiès, les éponges de Klein), à la projection ou à la coulure (Pollock ou Bram van Velde) par exemple, en fonction des propriétés de la matière mobilisée.

Ainsi, en même temps que l'œuvre sollicite un nouveau modus operandi, d'autres gestes que ceux du peintre et d'autres outils, l'œuvre d'art s'acquitte des prescriptions ontologiques du tableau qui reconduisent les exigences de la pratique picturale (tendre la toile sur un châssis, appliquer le pigment avec des pinceaux et des brosses). Simultanément, I'artiste semble renoncer à certaines responsabilités dans la praxis énonciative, qu'il cède à la matière elle-même, appelée à poursuivre sa propre énonciation. Ainsi les pigments extrêmement dilués de Bram van Velde prolongent-ils le geste du peintre tout en le contredisant par des coulures qui interviennent dans la forme tracée.

En interrompant une tradition picturale coutumière des pigments et des brosses et en renouvelant sans cesse les modalités de la rupture, de telles innovations esquissent un espace de liberté où les artistes s'efforcent de dégager le tableau de son ontologie.

\section{Comment la matière modifie le sens}

Ces premiers résultats tendent à révéler une autre acception de la matière qui, loin de se limiter à un statut de matériau non finalisé et inorganisé, renvoyant seulement à un point de vue épistémologique situé en amont des pratiques, s'impose comme une instance de renouvellement de la semiosis. Nous y reviendrons.

Mais il convient d'abord d'évoquer une possibilité de transformation du matériau pictural en matière, sans aucun apport hétérogène, mais du simple fait d'une densification du matériau pictural. Un tel phénomène se produit dans la peinture $d^{\prime}$ Eugène Leroy, dont nous avons déjà décrit le faire caractéristique. Dans ce cas, la superposition des couches permet d'épaissir le matériau pictural jusqu'à le «transcender » pour imposer le terme de matière, conçue dès lors comme signifié du matériau pictural.

Avec un peu d'attention, on peut apercevoir les conséquences d'une telle transformation sémantique qui permet de revisiter les propriétés du matériau pictural - en l'occurrence chromatiques, lumineuses et olfactives - pour produire de nouveaux effets de sens. En ce qui concerne le chromatisme, la superposition, aboutissant inévitablement au mélange des couleurs, tend à produire une invariable couleur marron dont ressortent seulement quelques points de lumière préservés par le mélange optique (BeyaertGeslin, 1998). Quant à l'incidence olfactive, elle tient à l'extrême concentration des effluves de la peinture à l'huile. Cette description pourrait sans doute se poursuivre avec intérêt et viendrait confirmer les modalités de la conversion esthésique; cependant, pour notre étude, il suffirait $d^{\prime}$ indiquer que la matière, devenue signifié du matériau pictural, s'affranchit alors des propriétés discrétisées du pigment pour exploiter sa propre richesse polysémique et imposer une présence polysensorielle convoquant d'autres alliances synesthésiques.

L'exemple des tableaux de Leroy amène à distinguer deux cas. Dans le premier, la matière est un composant hétérogène ajouté au matériau pictural; dans le second, elle résulte d'une transformation du matériau pictural lui-même par épaississement. Chaque fois, la matière $s^{\prime}$ impose comme un signifié auquel peuvent s'ajouter différents effets de sens intervenant à titre de connotation. L'efficience de tels effets de sens suppose toutefois que le peintre ait pris soin de préserver l'apparence triviale de la matière qui, pour intervenir dans la signification, doit être identifiée en tant que grandeur culturelle. Ainsi le sang ajouté aux peintures des Actionnistes viennois doit-il rester parfaitement reconnaissable pour que se révèle une relation au corps souffrant. De même, la révolution axiologique produite dans l'univers de 
valeurs des beaux-arts impose-t-elle que les excréments utilisés dans les œuvres de Gasiorowski ou Lizène soient identifiés comme tels.

\section{Matière et tridimensionnalité}

Un pas supplémentaire doit maintenant être fait. En effet, simplifiant à peine, nous pourrions affirmer que les enjeux de la matière passent nécessairement par l'introduction d'une troisième dimension. D'ailleurs, si les auteurs du Groupe $\mu$ (1992: 201) associent trois signifiés à la texture (la tridimensionnalité, la tactilomotricité et l'expressivité), la première est considérée comme son «signifié global majeur» et reliée à deux conceptions de la texture. Lorsque la troisième dimension est produite «directement», la texture est un grain, mais si elle est produite « de façon indirecte», c'est une macule. Selon le cas, une participation du corps ou de l'intellect sera requise, l'image saisie visuellement renvoyant «à une expérience tactile par une suggestion synesthésique», une participation différentielle qu'on traduirait volontiers par la valence de l'intensité (lorsque les effets de sens sont éprouvés par le corps, comme c'est le cas dans la peinture de Leroy), ou de l'étendue figurative (quand ces effets de sens renvoient à une représentation de l'objet par ses propriétés mimétiques).

Plus largement, les deux acceptions de la texture (grain ou macule) esquissent deux conceptions de la tridimensionnalité en tant que finalité de la pratique picturale. Comment la peinture représente-t-elle la troisième dimension? Une première acception envisage celle-ci comme une construction perspective alliant notamment les lois des perspectives géométrique et atmosphérique. Dans ce cas, elle est représentée de la même façon que la macule représente le grain et en rappelle l'expérience par des moyens synesthésiques. Une seconde acception envisage la troisième dimension en reproduisant son expérience, sur le modèle de la peinture de Leroy qui nous met en présence de la matière. Selon que la tridimensionnalité est représentée ou ostensive, les effets de sens renvoient donc à deux types d'expérience sensible dont un enjeu essentiel semble être la conversion du matériau pictural en tant que signifiant en une matière conçue comme signifié. Ainsi les paysages conformes aux exigences du genre, tel qu'il s'est autonomisé à la fin du $x v^{e}$ siècle, représentent-ils la spatialité par les moyens de la perspective, le matériau pictural tenant lieu pour ainsi dire de signifiant de la spatialité, tandis que les œuvres d'Anselm Kiefer entreprennent de représenter la spatialité naturelle par la densité qu'elle impose dans l'expérience du paysage, par des épaisseurs peintes ou constituées d'accumulations de feuilles de laurier odorantes qui associeront à l'effet de sens de spatialité la plénitude sensorielle de la nature.

Mais un commentaire s'impose. En effet, si la catégorie/texture versus matière/ suscite quelques réserves parce que la différence semble ne pouvoir être transformée en opposition, il convient pourtant d'opposer résolument la tridimensionnalité représentée et la tridimensionnalité ostensive qui ne peuvent collaborer à moins de pénaliser leur projet respectif. En effet, si nous entreprenons de représenter un paysage par les moyens de la perspective, la production d'une épaisseur matérielle ne peut que perturber la représentation de la tridimensionnalité, de même qu'à l'inverse la représentation de la troisième dimension contrevient au projet d'épaississement.

\section{Sémiotique de l'empreinte}

Ce rapport à la tridimensionnalité permet de situer les enjeux de la matière vis-à-vis d'un principe de concordance entre deux empreintes. Fontanille envisage une telle concordance entre une scène de production textuelle et une scène d'interprétation. Plaidant pour une sémiotique de l'empreinte, il note que celle-ci [...] prête attention au modus operandi de la production textuelle, tout autant qu'à celui de l'interprétation, car elle fait l'hypothèse que l'interprétation est une expérience qui consiste à retrouver les formes d'une autre expérience dont il ne reste que l'empreinte.

(2004 : 265)

Dès lors, poursuit l'auteur, il n'est plus de sémiotique «visuelle» ou «auditive» (visuel et auditif ne référant, en ce cas, qu'à un canal de réception), mais des "sémiotiques fondamentalement et irréductiblement synesthésiques». Sur ce principe s'impose notamment une correspondance entre les gestes qui «permettent de cerner et d'identifier la forme d'un objet» et ceux qui «étalent le pigment sur la toile pour y inscrire le modelé» (ibid.).

Si I'on suit cet auteur, l'empreinte picturale procède donc $d^{\prime}$ 'une correspondance entre deux scènes, ce qui permet de décrire les variations texturales sur la notion de rythme, de sorte que, un faire s'accordant à l'autre, les rythmes de la praxis énonciative renvoient à une autre expérience rythmique. Cependant, plutôt qu'une unique correspondance, il semble pertinent d'esquisser une double correspondance allant avec deux types de présence conformes à la dualité de la relation à la tridimensionnalité. Selon la structure du support, la densité des gestes, la qualité ${ }^{9}$ du matériau pictural et surtout selon sa quantité, une concordance différente s'établit avec le monde naturel qui modifie les termes 
de I'alliance synesthésique. En fonction de ces variables matérielles, la correspondance s'établit conformément au principe de représentation de l'expérience sensible, ce qui suppose un déploiement figuratif, ou conformément au principe de l'ostension fondé sur l'intensité. Dans un cas comme dans l'autre, la sémiotique synesthésique reste cependant de mise, car il s'agit toujours de renvoyer à une expérience faite par ailleurs, la différence entre les deux accords synesthésiques se laissant décrire à partir de la représentation/ostension, comme une différence de rythmes d'inscription des modalités sensibles.

\section{Claudel et la matière}

Sortir de la pratique picturale nous oblige à reconsidérer le problème. Il faut abandonner le principe du recouvrement, spécificité picturale, ce qui n'empêcherait pas de conserver l'idée que la matière se distingue du matériau pictural par une épaisseur, une densité permettant d'exploiter différents univers de sens. La matière se définit alors comme une épaisseur, une densité dont le faire artistique actualisera les propriétés dans la sémiose. Ce principe posé, il resterait à décrire ces potentialités de la matière qui déterminent le sens.

Une reprise d'un texte célèbre de Claudel (1946) consacré aux natures mortes hollandaises permet "d'entrer en matière». Celui-ci nous présente des «corps», soit des structures matérielles contenues dans une enveloppe et animées d'un mouvement, qui sont aussi des actants dotés de compétences modales, investis de valeurs et traversés par l'axiologie. L'écrivain observe tout d'abord la maigre variété de ce corpus, à peu près limité au matériel du repas eucharistique (du pain, du vin et un poisson), auquel il confère un contenu essentiellement symbolique. Selon lui, la présence fréquente d'un citron coupé en deux ou à demi pelé dont la spire pend au dehors et de toutes sortes de bols et d'assiettes, argumente cependant l'hypothèse d'une représentation du temps qui, outrepassant les possibilités du répertoire iconologique, passe par l'organisation des formes.

"La nature morte hollandaise est un arrangement qui est en train de se désagréger, c'est quelque chose en proie à la durée ", observe Claudel (1946: 48). Elle se compose donc toujours d' " un arrière-plan stable et immobile", composé de belles verreries bien droites, par exemple un long verre ou un calice, et «sur le devant toutes sortes d'objets en état de déséquilibre». Ainsi décrit-il une composition de l'arrière-plan vers I'avant-plan où un groupe rigide et érigé mais aussi figé par le temps s'oppose aux objets du devant, des végétaux abandonnés à leur propre gravité, en ébauchant une transformation aspectuelle. Si l'im- portance du contraste pour la signification se trouve ainsi soulignée ( « c'est l'immobilité quasi morale à l'arrière-plan, c'est cet alignement de témoins à demi aériens qui sur l'avant donne leur sens à tous ces éboulements matériels», précise l'auteur [ibid.]), l'intérêt de ces propositions réside dans le système semisymbolique élémentaire esquissé, où les contrastes du plan de I'expression arrière-plan/avant-plan, érigé/pendant, achronique/ chronique (ou immobile/mobile) et tension/laxité renvoient au plan du contenu à une transformation aspectuelle entre un avant et un après allant avec une transformation vie/mort. Ces propositions pourraient sans doute être généralisées au genre de la nature morte, valant aussi bien pour la nature morte française (Chardin) que pour les peintures de Claesz (Beyaert-Geslin, 2000), et révéleraient que les propriétés représentées signifient toujours relationnellement et topologiquement, en fonction de leur insertion dans un système semi-symbolique récurrent où I'alliance de la spatialité et de la temporalité dans l'aspectualité restitue une axiologie. On aperçoit ainsi un effet affectif qui, loin de résulter de la somme des tensions manifestées par les objets rassemblés, provient de la corrélation de ces tensions et, plus précisément, de l'orientation des valeurs tensives vers la désagrégation. En reliant I'arrière-plan et l'avant-plan du tableau, les «verreries» et "les objets en déséquilibre», l'érigé et le pendant, l'univers de la tension et celui de la laxité, le regard du spectateur actualise le mouvement organique qui va de la vie à la mort et met au jour la fragilité de la vie.

L'exemple des natures mortes hollandaises permet de montrer comment la mise en relation de matières aux propriétés opposées esquisse une dramaturgie, ajoutant ainsi son crédit à l'hypothèse d'une conception relationnelle et topologique de la signification. Bien qu'elle nous rattache à l'univers de la peinture, cette leçon nous sera utile pour aborder l'œuvre de Beuys car, au-delà des prescriptions du tableau, elle révèle que des corps, en l'occurrence des figures représentant des pots, des verreries ou un poisson, peuvent être investis de compétences actantielles.

\section{Beuys et la matière}

Beuys est un artiste majeur et extrêmement prolifique dont la production est estimée à plusieurs milliers d'œuvres. Si elle mérite cent fois qu'on s'y attarde, nous nous contenterons dans le cadre de cette étude de tirer quelques leçons de la matière, notion centrale en l'occurrence, infiniment thématisée pour rechercher $\mathrm{d}^{\prime}$ autres configurations sensibles ${ }^{10}$. Une célèbre déclaration de I'artiste témoigne d'ailleurs, mieux que tout autre commentaire, $d^{\prime}$ un souci de rupture avec les prescriptions ontologiques du 


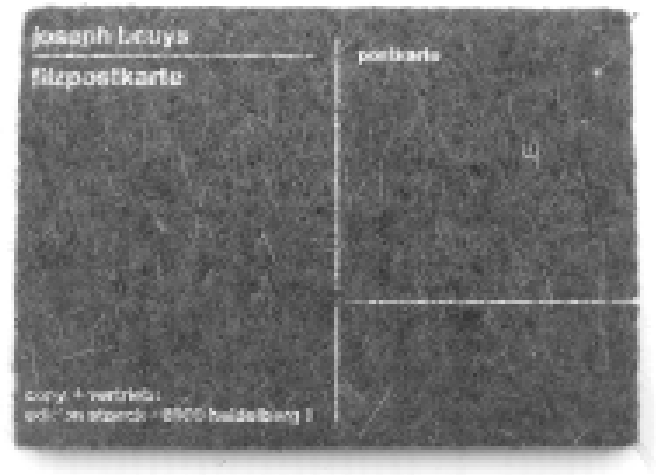

Joseph Beuys, Filzpostkarte, 1985. Sérigraphie sur feutre, 10,5 x 15 × 0,9 cm. Heidelberg, Éd. Staeck. Collection particulière.

tableau: «L'erreur commence quand on se met en tête d'acheter de la craie et de la toile» (nous traduisons) ${ }^{11}$.

L'œuvre de Beuys est essentiellement constituée, outre les dessins qui, eux-mêmes, renouvellent le matériau pictural par divers produits, d'installations mêlant les matériaux les plus variés et notamment le feutre et la graisse. Parce qu'ils dérogent aux prescriptions ontologiques des genres et témoignent d'un effort de rénovation d'une pratique artistique conçue dans son acception la plus accueillante, de tels matériaux se laissent décrire non pas comme des matériaux picturaux ou «sculpturaux», mais tout au plus comme des matériaux plastiques. Plus exactement, en s'affranchissant des prérogatives des genres et des pratiques, ils revendiquent l'appellation de matières et la référence à la matière. Inspirés par la mythologie personnelle de l'artiste et son sauvetage par les Tatars ${ }^{12}$, ceux-ci sont connotées symboliquement et engagent dès l'abord une axiologie ${ }^{13}$, mais leur richesse sensorielle et transformationnelle suffit à justifier leur importance dans la production de l'artiste autant que leur statut d'actant.

Tout d'abord, l'observation de cette œuvre permet de vérifier une ambiguïté particulièrement intéressante qui tient à la relation entre la chose et l'objet. Du point de vue de la philosophie des sciences et notamment de Dagognet (1989: 31) qui envisage le rapport chose/objet comme une opposition nature/culture, ces œuvres se laissent décrire telles des choses en raison de leur ancrage dans la nature, des choses cependant transformées en objets par le geste de l'artiste. Le point de vue sémiotique hésiterait de même entre les deux statuts, voyant déjà dans la matière le mouvement qui caractérise un objet et observant que le geste de I'artiste qui la découpe et la façonne permet aussi de la circonscrire dans une enveloppe. Mais si une règle générale veut que I'énonciation artistique procède à l'actualisation des propriétés $d^{\prime}$ 'une matière (chose) sous la forme d'un objet, l'exemple de
Beuys montre sa pertinence en révélant que l'effort de cet artiste vise parfois à estomper les contours de l'enveloppe, ce qui a pour effet de suspendre pour ainsi dire l'objectivation.

$C^{\prime}$ est un tel constat qu'imposent par exemple les «environnements » et les «blocks» disposés par Beuys lui-même dans le musée de Darmstadt, en Allemagne ${ }^{14}$. À peu près dénués de contour d'énoncé, les blocks se prolongent l'un dans l'autre en mêlant, dans la continuité $d^{\prime}$ 'une chose, un petit lapin en massepain, un tas de rognures d'ongles, des vitrines, des plaques de métal formant un dallage, le déroulé de feutre sur le sol ou son prolongement sur le mur qu'il recouvre comme la plus banale moquette. Les contours de l'œuvre étant estompés, celle-ci peine à s'accomplir en objet et se présente tel un assemblage de matériaux organiques qui préserve la continuité de la chose.

Cette hésitation (où est l'œuvre? où «s'arrête-t-elle» ?) due aux particularités de la situation énonciative n'oblitère aucunement le statut actantiel de la matière. Le feutre et la graisse se caractérisent par une extrême richesse sensorielle qui sature pour ainsi dire dès l'abord le plan de l'expression de l'œuvre. Ce sont à la fois des matières colorées identifiées par une couleur bien particulière (un certain jaune et un certain gris), des matières texturales et des matières aux propriété olfactives, thermiques ${ }^{15}$ voire auditives puisque le feutre assourdit les bruits 16 .

\section{Les compétences de la matière}

Dès l'abord, ces matériaux s'imposent tels des acteurs pourvus d'une identité modale singulière. À l'aune de la classification de Leroi-Gourhan, le feutre se définit comme un solide souple tandis que la graisse oscille plutôt entre le solide plastique susceptible « de pouvoir passer d'un état presque fluide à un état solide ou consolidé » (1992: 162) comme le précise cet auteur, et le fluide, catégorie très générale où il range aussi bien l'eau, le blé, les pommes que leurs contenants. À la différence des solides stables, I'un et I'autre sont donc susceptibles de se transformer en suivant un certain imaginaire modal, une «paléontologie du geste» (ibid.) assurant l'actualisation de leurs propriétés.

Le feutre provenant d'un solide fibreux d'origine naturelle et se présentant « en plaques ou en éléments allongés dont l'enchevêtrement assure la cohésion» (ibid.: 234), le geste énonciatif consistera à débiter, à coudre ou à superposer des plaques en suivant les potentialités de la matière. Sa qualité de solide souple le vouant à l'horizontalité, vectorialité caractéristique des œuvres de l'Informe ${ }^{17}$, le feutre réalisera donc sa vocation formelle qui est de suivre la gravité pour pendre, pour recouvrir ou constituer 
des couches. S'il se montre alors animé par une direction intentionnelle, c'est pourtant le geste énonciatif de l'artiste qui le met en action, révélant ainsi le mouvement de la matière comme principe même de l'intentionnalité ${ }^{18}$.

Comme le suggère I'hésitation entre le statut de solide plastique et de fluide, la direction intentionnelle de la graisse est plus ambiguë et esquisse une dramaturgie latente puisque les oeuvres sont susceptibles de changer de forme sous l'effet de la chaleur, au risque de compromettre leur intégrité d'objet. Ainsi une œuvre de Beuys faite de paquets de margarine emballés dans du carton, intentionnellement placée sous une verrière brûlante dans le cadre d'une exposition à Temse ${ }^{19}$, en Belgique, se transforma-t-elle en un parallélépipède de carton vide émergeant $d^{\prime}$ 'une mare jaune translucide.

Mais une telle aventure révèle une autre exigence de la graisse. Car lorsqu'il devient un fluide, sous l'effet d'une énergie, un solide souple réclame toujours un récipient, observe LeroiGourhan (1992: 207). Aussi la graisse requiert-elle un contenant, de même que la souplesse du feutre le voue à la pression de matières stables et pesantes, cuivre ou plomb, par exemple. Le mouvement de la matière induit alors une interaction avec $d^{\prime}$ autres matières agissant dans un rapport de forces, ce qui induit une inflexion du mouvement et une modification du schéma modal conforme à une structure passionnelle. Représentatives $d^{\prime}$ une telle interaction, les différentes versions de Fettstuhl (Chaise de graisse) sont constituées d'un siège dont l'angle est tapissé de margarine pour former un volume triangulaire. La graisse pouvant se transformer en fluide sous l'effet de la chaleur, la forme de l'œuvre de même que la semiosis restent instables et marquées par l'inaccompli. Dans ce cas, deux séquences canoniques caractérisent la structure modale de l'œuvre, selon que la direction de la matière est marquée par la tension ou la laxité, un événement de la matière permettant de basculer d'une situation énonciative à l'autre.

Cette description amène à réviser le rôle du geste et les présupposés de l'énonciation. En effet, comme nous l'avions constaté à propos des peintures de Bram van Velde, le geste qui actualise les propriétés de la matière n'assure pas nécessairement la stabilité de la forme énoncée qui, dans le cas de la graisse, peut à tout instant être mise en cause par le réchauffement. En pareil cas, on aperçoit une limitation de la responsabilité de l'artiste dans la praxis énonciative et une réévaluation de celle de la matière qui poursuit ou infléchit le cours de l'énonciation. En somme, si elle est caractérisée par certains /pouvoir faire/ et /devoir faire/, ces modalités se conçoivent à l'intérieur d'une structure modale com- plexe et argumentent une narrativité marquée par des séquences canoniques, des alternatives stratégiques et des bifurcations qui laissent la semiosis en suspens.

\section{Axiologie et moralité}

Mais la participation de la matière à l'énonciation ne s'arrête pas là. En tant qu'acteur, la matière est investie d'une identité culturelle particulière évoquant le nomadisme, le chamanisme, l'écologie, etc. Celle-ci renvoie en outre à un système de valeurs qui tend à inverser I'axiologie des beaux-arts par l'utilisation de matériaux triviaux ou mous, la mollesse modifiant le rapport à la gravité20 et introduisant un effet de sens dysphorique ${ }^{21}$. Investies d'une axiologie négative, les installations de Beuys se laissent ainsi décrire telles des natures mortes manifestant l'alliance paradoxale et caractéristique de la vie et de la mort ${ }^{22}$.

Cependant, pas plus que la signification des natures mortes hollandaises ne se résume à une iconologie, celle des œuvres de Beuys ne se limite à des assemblages d'objets ou aux propriétés des matières dont ils procèdent. Relationnelle et topologique, elle tient plutôt aux relations établies entre les objets, au contraste systématique du définitif et de l'éphémère, de l'érigé et du pendant, d'un contenant solide et d'un fluide, par exemple. II semble donc utile de reprendre les propositions de Claudel. Mutatis mutandis, la représentation étant convertie en ostension, on avancerait alors que la signification de ces œuvres tient à la relation établie entre les objets et les qualités, mais une différence essentielle s'imposerait alors. En effet, loin d'être discrètes et discrétisées par un tableau, les propriétés s'inscrivent dans la continuité, dans le mouvement de la matière en transformation qui les enchaîne et les scénarise. La signification ne tient pas au contraste d'un solide et d'un fluide mais à la transformation du solide en fluide et à la dramaturgie intime de la matière.

Il convient donc d'associer la signification à un double mouvement et à un double scénario aspectuelcorrespondant, pour l'un, à la direction intentionnelle d'une matière aux multiples propriétés et, pour l'autre, à la dégradation organique qui accompagne la matière vers la mort et l'insignifiance ${ }^{23}$. Ainsi le mouvement actantiel manifeste-t-il, de deux façons, la moralité de la matière.

Toutefois, une ultime leçon s'impose. En effet, si la praxis énonciative est une actualisation du mouvement de la matière qui s'énonce en même temps qu'elle devient objet, accompagner ce mouvement jusqu'à son épuisement revient à perdre l'objet pour revenir à la chose, à l'indistinct et à l'insignifiance. Le mouvement d'énonciation conduit alors à une sorte de désé- 
nonciation. Autrement dit, la décomposition organique de la matière est aussi une décomposition esthétique.

\section{NOTES}

1. Les textes du recueil ont été publiés entre 1922 et 1947.

2. Cette conception rejoint la conception de Manzini pour qui la différence entre matière et matériau tient au fait que le second s'intègre «dans un projet et devient composante d'un produit» (1989: 14).

3. À propos de la peinture cubiste, nous avions montré comment deux systèmes entrent en rivalité, celui de l'écriture et celui de la peinture, et comment ils occasionnent une négociation stratégique entre le support formel et le support matériel. Voir à ce sujet Anne Beyaert-Geslin (2005). 4. La démarche de support surface se laisse également décrire comme une thématisation méthodique des propriétés du support matériel, mais elle ne pourrait être considérée comme exemplaire dans la mesure où ce projet aboutit en certains cas à ne conserver que les éléments du support (une toile, un cadre...) en virtualisant la peinture elle-même.

5. Beaucoup d'études sémiotiques ont néanmoins étudié le tableau ou la photographie en les considérant comme des images de livres, sans tenir compte de la dimension texturale, voire en éludant la question du format, ce qui virtualise le corps de l'observateur.

6. Il faudrait bien sûr tenir compte, par exemple, des différences de coloration des reproductions.

7. Greenberg définit l'avant-garde par le fait qu'elle se spécialise sur ellemême: «L'artiste ou le poète d'avant-garde essaie en fait d'imiter Dieu, en créant quelque chose qui ne vaille que par soi-même, à la manière dont la nature est valide, dont un paysage - pas son image - est spécifiquement valide: c'est-à-dire quelque chose de donné, d'incréé, libre de toute signification, de toute ressemblance, de tout modèle » (1989: 12). Plutôt que Dieu, l'artiste imite "les modes et les procédures artistiques et littéraires eux-mêmes », précise-t-il, en se concentrant sur « les moyens de sa pratique». Cette conception doit bien entendu être confrontée à celle de Goodman pour qui une représentation, loin d'être un enregistrement naif du monde, restitue toujours un certain rapport au monde. Cette proposition infléchitl'affirmation de Greenberg en faisant de la composante métadiscursive non pas une particularité de l'œuvre d'avant-garde mais l'apanage de toute représentation.

8. Une texture est une microtopographie qui reçoit le statut de texture en raison d'une loi de répétition (Groupe $\mu, 1992: 70)$. «C'est le rythme qui fait la texture» (ibid. : 199).

9. Selon Fontanille (2004: 265), la présence assurée par l'empreinte ne connaît que quatre variables : 1 ) la structure matérielle du support; 2 ) le type d'événement, de geste ou de technique qui assure l'inscription; 3) l'intensité et l'étendue de ces derniers; 4) la densité et la quantité des correspondances. Je remercie Maria Giulia Dondero qui m’a indiqué cette référence.

10. Parmi bien d'autres critiques, De Meredieu (1994: 17) souligne l'importance de la matière chez Beuys: « Jamais les sens n'ont été amenés à buter à ce point sur une matière $[. .$.$] nous sommes renvoyés à$ l'archaïsme d'une matière sourde, qui ne se répand pas en reflets, qui occulte donc le système de la représentation. Clos, fermé sur ses secrets, d'une résonance purement interne, le matériau possède ici l'équivalent des propriétés de l'inconscient. Tout se passe comme si le discours de la matière ainsi que l'ensemble des échos qu'elle génère étaient renvoyés dans une dimension intérieure ».

11. «Der Fehler fängt schon an wenn einer sich anschickt, Kreide und Leinwand zu kaufen». L'affirmation figure sur une carte postale des éditions Staeck.

12. Cette mythologie s'inscrit dans un épisode central. D'abord berger en Allemagne, Beuys fut pilote de bombardier dans la Luftwaffe lors de la Seconde Guerre mondiale. Son avion s'écrasa en Crimée en 1942 et le soldat, à moitié gelé, fut recueilli par une tribu de Tatars qui le ramenèrent à la vie en le couvrant de leurs traditionnelles couvertures de feutre et en le réchauffant avec de la graisse animale avant de l'admettre comme un des leurs. Maintes fois évoqué, l'épisode est relaté notamment par Alain Borer (1994: 14). Si la véracité a été contestée, elle donne consistance à la figure du chaman chère à Beuys, sorte de passeur entre la nature et la culture. Jan Hoet assure par exemple: «Joseph Beuys envisage son art comme la recherche d'un état originel, seul état où l'homme, les animaux et la nature se savent unis de manière organique» $(1987: 77)$.

13. Pour l'artiste, ces matériaux comportent des «traits chamanistiques initiatiques», comme l'indique Bernard Lamarche-Vadel (1985: 90).

14. On pourra se reporter à Ammann et Präger (1991).

15. Elles sont censées avoir réchauffé Beuys en Crimée.

16. Nous renvoyons à la visite de Plight (1985), étrange installation polysensorielle du Musée national d'art moderne, un habitacle tapissé de rouleaux de feutre et accueillant un piano, dont les propriétés ont été habilement thématisées en une sorte de chaîne de sensations où la couleur s'allie à la chaleur, à l'odeur puis au silence.

17. Nous renvoyons pour une anthologie de l'Informe à Bois et Krauss (1996).

18. La direction intentionnelle que nous associons à la matière rejoint, pour les arts plastiques, les conceptions de Focillon (1943) pour qui la matière, à la fois structure et action, possède une vocation formelle.

19. Voir le catalogue de l'exposition Ponton (23 juin au 30 septembre 1990), Gent, Museum van Hedendaagse Kunst.

20. Pour mesurer la rupture axiologique et sémantique que représente I'orientation descendante ou horizontale, on serait tenté d'évoquer la perspective édifiante de l'art dont Fréchuret (1993) situe les prémices au XVIII siècle et l'apogée au XIXe. Lorsque la sculpture part du sol, elle illustre, aux dires de cet auteur, le sens métaphorique d'édifier, un terme d'architecture qui revêt un sens moral pour signifier former, instruire, éclairer, porter à la vertu ou à la piété.

21. À propos des œuvres pendantes d'Eva Hesse, nous avons évoqué un effet de sens de fragilité, voire de morbidité (Beyaert-Geslin, 2003b).

22. Dans la plupart des langues, la nature-morte (Stilleben, still life, stilleven) est un oxymoron qu'on trouve dès les noms originels du genre (vie coye, par exemple). Le terme est toujours un nom adjectivé et l'adjectif vient contredire le sens du substantif.

23. Une visite au musée de Darmstadt suffit à révéler le sort funeste des rognures d'ongles, des petits lapins de massepain, des saucissons, de la chaise de graisse et des rouleaux de feutre insidieusement minés par la poussière et entraînés peu à peu vers la mort, l'indifférence de la matière et la perte sémantique. 


\section{RÉFÉREN CES BIBLIO G RAPHIQ U ES}

AmmanN, J. C ET C. PrÄGER [1991]: Museum für Moderne Kunst und Sammlung Ströher, Francfort-sur-le-Main, Musée d'art moderne.

BeYAeRT-GeSLIN, A. [1998] : "Plasticité et signification: le cas d'Eugène Leroy ", Protée, vol. 27, n², 125-131;

[2000]: «Chardin: un lapin, deux grives et quelques matériaux

sémiotiques", notes d'exposition, Nouveaux Actes Sémiotiques nos 68, 69, 70, Limoges, PULIM, 144-149;

[2003a] : «Brancusi, la ressemblance et le rythme», Protée, vol.

$31, n^{\circ} 2,73-81$;

[2003b]: «Organique et méta-sémiotique», Modèles linguistiques, tome XXIV, vol. 47, 57-74.

[2003c]: «Texture, couleur, lumière et autres arrangements de la perception ", Protée, vol. 31, no 3, 81-90;

[2005]: «La typographie dans le collage cubiste: De l'écriture à la texture ", dans M. Arabyan et I. Klock-Fontanille (dir.), L'Écriture entre support et surface, Paris, L'Harmattan, 131-152;

[2008]: "La figure, le fond, le gouffre», Actes du colloque Groupe $\mu$, soixante ans de recherche collective (à paraître).

BOIS, Y.-A. et R. KRAUSS [1996] : L'Informe, mode d'emploi, catalogue d'exposition, Paris, Éd. du Centre Georges-Pompidou.

BORDRON, J.-F. [2003] : "La signification des objets », dans F. Parouty et

C. Zilberberg (dir.), Sémiotique et Esthétique, Limoges, PULIM, 469-488.

BORER, A. [1994]: Déploration de Joseph Beuys, catalogue du Musée

national d'art moderne, Paris, Éd. du Centre Georges-Pompidou.

CLAuDEL, P. [1946] : L'œil écoute, Paris, Gallimard.

Dagognet, F. [1989] : Éloge de l'objet. Pour une philosophie de l'objet,

Paris, Vrin.

DE MEREDIEU, F. [1994] : Histoire matérielle et immatérielle de l'art, Paris,

Bordas.
Dubuffet, J. [1973] : L'Homme du commun à l'ouvrage, Paris, Gallimard. FLOCH, J.-M. [1985] : Petites Mythologies de l'œil et de l'esprit. Pour une sémiotique plastique, Paris, Hadès-Benjamins.

FOCILLON, H. [(1943) 2000]: Vie des formes, Paris, PUF.

FonTANILLE, J. [2004] : Soma et Séma. Figures du corps, Paris, Maisonneuse et Larose;

[2005]: «Du support matériel au support formel», dans M. Arabyan et I. Klock-Fontanille (dir.), L'Écriture entre support et surface, Paris, L'Harmattan, 183-200.

FRÉCHURET, M. [1993] : Le Mou et ses formes. Essai sur quelques catégories de la sculpture du vingtième siècle, Paris, École nationale supérieure des beaux-arts.

GoOdman, N. [1968]: Langages de l'art, trad. de J. Morizot, Paris, Hachette.

GREENBERG, C. [(1988) 1989] : «Avant-garde et kitsch», Art et culture Essais critiques, trad. de Ann Hindry, Paris, Macula, 9-28.

Groupe $\mu$ [1992] : Traité du signe visuel. Pour une rhétorique de l'image, Paris, Seuil.

HOET, J. [1987] : «Dessins: un état originel», Artstudio, n 4 (Spécial Joseph Beuys), 76-83.

KLEE, P. [(1956) 1999] : Théorie de l'art moderne, trad. de P.-H. Gonthier, Paris, Gallimard, coll. «Folio essais".

LAMARCHE-VADEL, B. [1985] : Joseph Beuys, is it about a bicycle?, Paris, Marval, coll. "Galerie Beaubourg », Vérone, Sarenco-Strazzer.

Lerol-Gourhan, A. [(1971) 1992] : L'Homme et la Matière, Paris, Albin Michel.

MAnZINI, E. [1989]: La Matière de l'invention, Paris, Éd. du Centre Georges Pompidou/CCl.

MOHOLY-NAGY, L. [(1993) 2007]: Peinture, photographie, film et autres écrits sur la photographie, Paris, Gallimard. 